\title{
Changes in Social Function as a Trigger to Screen for Cognitive Impairment
}

\author{
Rafael D. Romo, PhD, RN, $P H N^{1,2}$ and Sei J. Lee, MD, MAS $S^{1,2}$ \\ 'Geriatrics, Palliative and Extended Care Services, San Francisco Veterans Affairs Medical Center, San Francisco, CA, USA; ${ }^{2}$ Division of Geriatrics, \\ University of California, San Francisco, San Francisco, CA, USA.
}

J Gen Intern Med 31(8):826-7

DOI: $10.1007 /$ s11606-016-3755-6

(C) Society of General Internal Medicine 2016

$\mathrm{T}$ he onset of cognitive decline is often a tumultuous time for patients and families. Older adults, fearing a loss of independence, frequently deny they are having problems and actively hide evidence of it. This, in turn, puts patients at risk for injury from accidents. Patients and families often recognize that something is amiss, but cannot identify the problem. This uncertainty leads to conflict and stress between patients and their families, increasing caregiver burden and the potential for domestic violence.

Consequently, understanding the risk factors for mild cognitive impairment and identifying interventions to improve quality of life and reduce caregiver burden is of immense importance to the care of older adults. The current evidence, however, focuses primarily on those with advanced dementia and Alzheimer's disease, while much less is known about the impact of mild cognitive impairment on older adults' health, social relationships, and quality of life.

\section{SOCIAL FUNCTION AND MILD COGNITIVE IMPAIRMENT}

Kotwal and colleagues ${ }^{1}$ have taken an innovative and significant step toward filling this gap in understanding. In their report published in this issue of JGIM, they specifically examine the relationships between mild cognitive impairment, multiple facets of social functioning, and gender. Using a nationally representative sample of community-based older adults (Wave 2 of the National Social Life, Health, and Aging Project), the researchers examined respondent data, and classified participants as having normal cognition, mild cognitive impairment, or dementia, using the Montreal Cognitive Assessment. The authors found that those with dementia had smaller,

Published online May 31, 2016 denser social networks, perceived less social support, and were less engaged in the community, and that these differences were also present among those with mild cognitive impairment, indicating that even early changes in cognition may disrupt social functioning. In addition, the researchers found that despite the evidence that women are better at mobilizing social support for themselves, women with cognitive impairment perceived less social support compared to men. Men with cognitive impairment felt they received more support from their spouses and socialized more with friends and neighbors than did women.

\section{IMPLICATIONS}

These findings have several important implications. Though some clinicians believe that there are few effective interventions for patients with cognitive impairment, ${ }^{2}$ a variety of interventions have been shown to improve outcomes in patients with cognitive impairment. First, Kotwal and colleagues point to evidence of the protective nature of social function on developing dementia. Furthermore, interventions focused on maintaining and improving interpersonal relationships and communication among patients with dementia and mild cognitive impairment have been shown to improve quality of life and reduce caregiver burden. ${ }^{3}$ Second, memory training and cognitive behavior therapy have been found to improve overall cognitive functioning in patients with mild cognitive impairment. ${ }^{4}$ Third, organized exercise programs in patients with dementia or mild cognitive impairment can improve both cognitive and physical functioning. ${ }^{5,6}$

US Preventive Services Task Force guidelines currently do not recommend routine screening for cognitive impairment in the absence of symptoms because the "evidence is insufficient to assess the balance of benefits and harms of screening." ${ }^{7}$ However, the present study suggests that our conceptualization of the symptoms of cognitive impairment may be too narrow, as the results show that disrupted social functioning may be an early symptom of cognitive decline that merits additional testing. Screening 
among these patients is likely to have a higher yield, making it more likely to benefit patients. For patients and their families, simply knowing there is an explanation for the difficulties they are experiencing can alleviate stress and reduce conflict. Thus, although it is currently unclear whether routine screening for cognitive impairment would be helpful, this study raises the possibility that in patients with impaired social functioning, screening for cognitive impairment may convey benefits.

The use of impairments in social functioning as a trigger for cognitive screening has the potential to help a significant number of older adults who have undiagnosed mild cognitive impairment or dementia. Kotwal found that $26 \%$ of the sample had mild cognitive impairment and $15 \%$ had dementia, which is similar to prevalence rates observed in prior studies. ${ }^{8}$ Since mild cognitive impairment is often undiagnosed ${ }^{9}$ and interventions can improve patient and caregiver well-being, using impairments in social functioning to trigger cognitive screening holds the potential to improve patient and caregiver outcomes.

\section{NEXT STEPS}

What Kotwal and colleagues have done is to highlight how much is unknown in this arena. Indeed, we have limited research examining the changes in social functioning as a patient progresses from mild cognitive impairment to dementia. As a consequence, we do not really know the full impact of the changes in social functioning that occur across the spectrum of cognitive impairment in relation to the quality of life for patients and the caregiving burden for families. We have even less evidence on which interventions maximize quality of life in patients across the spectrum of cognitive impairment. Subsequent research should focus on confirming and more fully elucidating the complex relationship between social functioning and cognitive impairment, with an eye toward potential interventions that may improve outcomes in patients with mild cognitive impairment or dementia.

Corresponding Author: Rafael D. Romo, PhD, RN, PHN; Geriatrics, Palliative and Extended Care ServicesSan Francisco Veterans Affairs Medical Center, 4150 Clement Street (181G), San Francisco, CA 94121, USA (e-mail: Rafael.Romo@ucsf.edu).

\section{Compliance with ethical standards:}

Conflict of interest: The authors report no conflict of interests.

\section{REFERENCES}

1. Kotwal AA, Kim J, Waite L, Dale W. Social function and cognitive status: results from a US nationally-representative survey of older adults. J Gen Intern Med. 2016. doi:10.1007/s11606-016-3696-0.

2. Martin S, Kelly S, Khan A, Cullum S, Dening T, Rait G, Fox C, Katona C, Cosco T, Brayne C, Lafortune $\mathbf{L}$. Attitudes and preferences towards screening for dementia: a systematic review. BMC Geriatr. 2015;10:66.

3. Lu YYF, Ellis J, Yang Z, Weaver MT, Bakas T, Austrom MG, Haase JE. Satisfaction with a family-focused intervention for mild cognitive impairment dyads. J Nurs Scholarsh. 2016.

4. Li H, Li J, Li N, Li B, Wang P, Zhou T. Cognitive intervention for person with mild cognitive impairment: a meta-analysis. Ageing Res Rev. 2010;10:285-296.

5. Barnes DE, Santos-Modesitt W, Poelke G, Kramer AF, Castro C, Middleton LE, Yaffe K. The Mental Activity and eXercise (MAX) trial. A randomized controlled trial to enhance cognitive function in older adults. JAMA Intern Med. 2013;179:797-804.

6. Hagovská M, Olekszyová Z. Impact of the combination of cognitive and balancing training on gait, fear and risk of falling and quality of life in seniors with mild cognitive impairment. Geriatr Geront Int. 2015.

7. US Preventative Services Task Force. Cognitive impairment in older adults: Screening. http://www.uspreventiveservicestaskforce.org. Accessed April 28, 2016.

8. Plassman BL, Langa KM, Fisher GG, Heeringa SG, Weir DR, Ofstedal MB, Burke JR, Hurd MD, Potter GG, Rodgers WL, Steffens DC, McArdle JJ, Willis RJ, Wallace RB. Prevalence of cognitive impairment without dementia in the United States. Ann Intern Med. 2008;148:427-434.

9. Chodosh J, Petitti DB, Elliot M, Hays RD, Crooks VC, Reuben DB, Buckwalter JG, Wenger N. Physician recognition of cognitive impairment: evaluating the need for improvement. J Am Geriatr Soc. 2004;52:1051-1059. 